\title{
Reseacrch Suatere \\ Polyostotic fibrous dysplasia complicated by pathological fracture of right femoral shaft with nonunion-A Case Report
}

Zhu Dong ( $\nabla$ zhu_dong@mail.jlu.edu.cn )

First Hospital of Jilin University

Yang Qifan

First Hospital of Jilin University

Liu jing

Binzhou Medical College Hospital

Tan lei

First Hospital of Jilin University

Jiang ye

First Hospital of Jilin University

\section{Research Article}

Keywords: Fibrous dysplasia, Shepherd's crook deformity, Nonunion, Osteotomies, Femoral shaft fracture

Posted Date: February 24th, 2022

DOI: https://doi.org/10.21203/rs.3.rs-1367863/v1

License: (c) (1) This work is licensed under a Creative Commons Attribution 4.0 International License. Read Full License 


\section{Abstract}

Introduction: Fibrous dysplasia is a benign fibrous bone tumor that accounts for $5 \%$ to $10 \%$ of benign bone tumors. It can manifest as simple fibrous dysplasia ( $70 \%-80 \%)$, polyostotic fibrous dysplasia (20\% -30\%), with approximately the same incidence in men and women(1). We report a patient with a rare case of multiple fibrous dysplasia combined with proximal femoral shepherd deformity with pathological fracture of the femoral shaft complicated by nonunion. It is necessary to understand the disease in more detail to avoid overtreatment of benign lesions or misdiagnosis of malignant tumors and other diseases.

Case presentation: A 58-year-old man with polyostotic fibrous dysplasia, bilateral proximal femur deformity, Shepherd's angle deformity, right femoral shaft pathological fracture complicated by nonunion, we under fluoroscopy, in the obvious proximal fracture, take osteotomy, and process the shape of the cut bone fragment to adapt it to the corrected force line, and then restore it back to its original position, using intramedullary nailing technology complete the operation.

Three months after the operation, he came to the hospital for re-examination, and an X-ray of the right femur was taken. It was found that the fractured end had a tendency to heal. The patient was instructed to gradually bear weight. After six months of re-examination, the patient could walk with a walker. One year after the operation, the patient could walk without a walker and take care of himself at home. However, there was still stretch-like pain in the right lower back, but it was tolerable.

Conclusions: For patients with polyostotic fibrous dysplasia combined with proximal femoral shepherd deformity and pathological fracture of the femoral shaft with nonunion, osteotomy combined with intramedullary nailing is a simple and convenient way to correct the deformity and obtain correct fracture alignment.

\section{Introduction}

The concept of "fibrous dysplasia" was introduced by Lichtenstein in 1938(2), which is a benign fibrous bone tumor in which fibrous tissue replaces normal bone tissue with metaplastic young woven bone. It accounts for about 5 to 10 percent of benign bone tumors. It can present as simple fibrous dysplasia (70\% - 80\%), polyostotic fibrous dysplasia $(20 \%-30 \%)$, Mccun-Albright syndrome $(2 \%-3 \%)$, or Mazabraud syndrome(fibrous dysplasia with intramuscular myxoma) (3). In 1891, von Recklingosen first described that the proximal femur is one of the most common sites. Mechanical stress and repeated fractures result in progressive varus and bowing, typical of shepherd's curvature (4). Shepherd's deformity is a deformity that develops through osteoporosis and constant microfracture and fracture repair and is associated with limb pain, lameness, and femoral neck fractures (5)

A classification of proximal femoral deformities has previously been proposed based on the largest cohort of patients with fibrous dysplasia known to us. It is divided into six types according to the neck axis angle and the presence or absence of side axis bending. Types 1 and 2 deformities usually do not progress, while types 3-6 deformities have a higher recurrence rate even with treatment. Type 3 can be 
corrected with an intertrochanteric osteotomy; types 4 and 5 can be corrected with one or more osteotomies, and type 6 can be fixed with one or more femoral intertrochanteric osteotomies. Osteotomy to correct the deformity. Whenever possible, all associated deformities should be treated with appropriate osteotomy (4) It has also been suggested that polyostotic fibrous dysplasia imaging features at least one of the following: (a) a typical "ground glass" appearance, (b) radiographic Increased or decreased density, (c) bone cyst, or (d) enlarged and/or irregular femoral profile; the presence and extent of the axial cortex and medullary canal (6) consistent with the disease based on the patient's imaging profile Performance.

Regarding the treatment of fibrous dysplasia, there are many treatment options. Initially, there were simple curettage and bone grafting to treat related deformities. Later, related treatment methods such as osteotomy, plate fixation, and intramedullary nailing appeared $(7,8)$.

We report a rare case of multiple fibrous dysplasia combined with proximal femoral shepherd deformity in a patient with a pathological fracture of the femoral shaft with nonunion. As far as we know, this is a rare case in China. By providing this case and treatment, we can provide reference experience for medical workers to understand, diagnose and treat this disease in the future.

\section{Case Report}

A 58-year-old man developed bilateral lower extremity deformities when he was young. He developed pain in his right thigh with no obvious cause 8 years ago. He was still able to farm daily and did not receive special treatment. The pain in the right thigh worsened 3 months ago and he was unable to stand immediately. He went to a local hospital and was diagnosed with a fractured right femoral shaft. No surgical treatment was performed, but conservative treatment was given at home. 2 months ago, consciously improved and walked down the ground, fell again, and the pain in the right thigh worsened. Conservative treatment was given at home again. Recently, he was admitted to our hospital because of worsening pain.

On admission, the clinical presentation was consistent with varus hip deformity, possibly with bilateral femoral neck deformities (Fig. 1). In line with Tscherne type I closed fracture soft tissue injury, the remaining manifestations did not have any endocrine disturbance, pigmentation changes, or precocious puberty changes. Both lower extremities were deformed, and both knee joints were huge (Fig. 2). In view of the abnormal bone quality of the patient, the imaging examination showed: multiple fibrous dysplasia combined with proximal femoral shepherd deformity, pathological fracture of the femoral shaft complicated by bone Nonunion (Fig. 3), multiple fracture lines can be seen on the right femur, old fracture lines and new fracture lines coexist, and the original fractures are malunion. The fractured end of the new fracture was obviously displaced. The patient's hemoglobin, total number and classification of white blood cells, erythrocyte sedimentation rate, C-reactive protein, calcium, phosphorus, alkaline phosphatase and other blood and serum biochemical indicators and hormone levels were within the normal range. On the second day of hospitalization, the right femoral shaft fracture was treated with open reduction, 
intramedullary nailing, internal fixation and osteotomy under general anesthesia, and the deformity was corrected and the fracture was fixed in one stage.

According to the length of the unaffected limb (Fig. 4), a special length of intramedullary nail is ordered. The patient is placed in the supine position and placed on the fluoroscopic bed. After the general anesthesia takes effect, the affected limb is adducted to facilitate the opening of the surgical approach. A longitudinal incision is made in the middle of the lateral thigh. about $10 \mathrm{~cm}$. The incision is slightly longer proximal to the fracture and shorter distally. Cut the skin, subcutaneous tissue and fascia lata, bluntly separate the vastus lateralis muscle, expose the fracture end, remove the accumulated blood and granulation tissue, cut the bone at the most obvious deformity of the proximal femur, correct the original deformity, protect the severed bone, and use the osteotome was trimmed to remove the obvious bending of the proximal and distal bones (as far as possible to ensure that the course of the medullary canal is consistent with the physiological curvature of the femur); the gold finger was inserted retrogradely at the proximal end of the new fracture, and trochanter of femur was pierced under C-arm fluoroscopy. After the guide wire is pierced through the trochanter, the trimmed bone segment clamp is reset to its original position, the guide wire is passed through and led to the distal end, and the specific intramedullary the nail was inserted anterogradely into the medullary cavity along the guide wire, and a blocking screw was drilled into the anterior side of the distal end of the femur. The intramedullary nail was slowly passed under the blocking screw, and the bone defect was filled with bone graft. The intramedullary nail was seen under $\mathrm{C}$-arm fluoroscopy. The position is good, the alignment of the fracture end is good, the femoral deformity is corrected, Through the guide, place two screws $5 \mathrm{~mm}$ below the femoral head through the femoral neck, the fracture end is compressed, and the fixation is firm, the position is good; after that, three screws are fixed at the distal end of the intramedullary nail, and the blocking screw is removed. Another fluoroscopy showed that the fracture was well reduced, the alignment was good, the deformity was corrected, and the internal fixation was firm. The operation was completed (Fig. 5). The patient was safely returned to the ward after the operation. On the third day after the operation, he reported that the right psoas muscle was tense, showing traction-like pain. After physical examination, it was found that the psoas muscle was tense and tenderness was positive. He was given hot compress treatment, and the symptoms were relieved. After the operation, the deformity of the right thigh of the patient was relieved, but the knee valgus was still present (Fig. 6).

Three months after the operation, he came to the hospital for re-examination. An X-ray of the right femur was taken. It was seen that the fracture end had a tendency to heal, and the patient was instructed to gradually bear weight (Fig. 7); re-examination six months after the operation, the right femur was examined on the front and lateral sides, and the fracture was healed well. Now the patient can walk with the aid of a walker (Fig. 8); One year after the operation, the X-ray of the right femur was taken on the front and the lateral side (Fig. 9): it was seen that the fractured end had healed. Now the patient can walk without the aid of a walker and can take care of himself at home (Fig. 10 Fig. 11). However, there was still stretch-like pain in the right lower back, but it was tolerable. This case report has been verbally agreed by the patient and his family (video attached at the end of the article). 


\section{Discussion}

The concept of "fibrous dysplasia" was introduced by Lichtenstein in 1938(2), which is a benign fibrous bone tumor in which fibrous tissue replaces normal bone tissue with metaplastic young woven bone. It accounts for about 5 to 10 percent of benign bone tumors. It can present as simple fibrous dysplasia (70\% - 80\%), polyostotic fibrous dysplasia $(20 \%-30 \%)$, Mccun-Albright syndrome $(2 \%-3 \%)$, or Mazabraud syndrome (fibrous dysplasia with intramuscular myxoma) (3). Of the affected sites, the proximal femur is one of the most common. As a rare skeletal disease, some scholars believe that its pathogenesis is that the mutation of somatic activation cam regulatory protein Gsa causes normal bone and bone marrow to be replaced by abnormally proliferated osteogenic precursors, resulting in deformity, fracture, dysfunction and pain. The disease can lead to a significant decrease in the patient's quality of life.

Nonunion is a rare disease in clinical practice. Once it occurs, it can cause huge living and economic burdens to patients and their families. At present, there is no consensus among countries on the definition of nonunion; the US Food and Drug Administration (FDA) defines the disease as: no healing on imaging for 9 months and no significant progress in healing in the past 3 months (9). The Danish Orthopedic Trauma Society defines nonunion as "a fracture that does not heal without further intervention" (10). By consulting relevant information, Protein antibodies, PTH and PTHrP have therapeutic effects on it, but there is no definite clinical trial to prove this conclusion(6); the most widely accepted treatment is still surgery. To strong fixation, restore the stability of the fracture-based.

Regarding the different manifestations and different stages of shepherd's flexion deformity, it is the most sensible choice to take a targeted treatment method. No matter which customized method is chosen, it is the most important to achieve the correct alignment.

In the past, doctors have used zoledronic sodium + Kirschner wire fixation, DHS, locking plate and other treatment methods for patients with multiple fibrous dysplasia combined with pathological fractures. In the author's opinion, the use of intramedullary nails can effectively fix the fracture site, provide a relatively stable fracture healing environment, and increase the chance of pathological fracture healing. Therefore, we believe that intramedullary nailing is a practical, effective and simple method to correct the pathological curvature of the proximal femur. Furthermore, this allows for faster treatment and recovery with a lower cost of surgery, which met the primary requirements of the patient in this case.

In summary, we report a rare case of multiple fibrous dysplasia with proximal femoral shepherd deformity with a pathological fracture of the femoral shaft with nonunion. In this patient's operation, we successfully reduced the fracture and achieved healing through osteotomy and intramedullary nailing, which effectively improved the patient's prognosis and saved the patient a lot of treatment costs. This will provide a reference for future clinical diagnosis and treatment of patients with similar conditions.

\section{Declarations}


The author agrees to publish this article.

\section{Statement of Informed Consent】}

Informed consent was obtained from the patients in this case study.

\section{Conflict of interest:}

None

\section{Source of support:}

This study was supported by the National Natural Science Foundation of China (Grant No12072129).

\section{Authors' contributions:}

Professor Dong Zhu and Professor Lei Tan are responsible for diagnosing diseases and reviewing articles, Qifan Yang is responsible for editing articles, Jing Liu is responsible for contacting patients for review and collecting imaging data, and Ye Jiang is responsible for editing articles

\section{References}

1. Nishiwaki T, Uchikawa S, Kusakabe H, Seki A, Eguchi Y, Takayama S, et al. Pronounced femur malunion after pathological bone fracture due to a simple bone cyst in the shaft of the femur, treated using Ilizarov fixation: a case report. J Med Case Rep. 2018;12(1):173.

2. GARLOCK JH. The differential diagnosis of hyperparathyroidism with special reference to polyostotic fibrous dysplasia. Ann Surg. 1938.

3. Muthusamy S, Subhawong T, Conway SA, Temple HT. Locally aggressive fibrous dysplasia mimicking malignancy: a report of four cases and review of the literature. Clin Orthop Relat Res. 2015;473(2):742-50.

4. Ippolito E, Farsetti P, Boyce AM, Corsi A, De Maio F, Collins MT. Radiographic classification of coronal plane femoral deformities in polyostotic fibrous dysplasia. Clin Orthop Relat Res. 2014;472(5):155867.

5. Al-Mouazzen L, Rajakulendran K, Ahad N. Fibrous dysplasia, shepherd's crook deformity and an intracapsular femoral neck fracture. Strategies Trauma Limb Reconstr. 2013;8(3):187-91. 
6. Kostenuik P, Mirza FM. Fracture healing physiology and the quest for therapies for delayed healing and nonunion. J Orthop Res. 2017;35(2):213-23.

7. Enneking W, Gearen P. Fibrous dysplasia of the femoral neck. Treatment by cortical bone-grafting. The Journal of bone and joint surgery American volume. 1986;68(9):1415-22.

8. Stephenson R, London M, Hankin F, Kaufer H. Fibrous dysplasia. An analysis of options for treatment. The Journal of bone and joint surgery American volume. 1987;69(3):400-9.

9. The differential diagnosis of hyperparathyroidism with special reference to polyostotic fibrous dysplasia.

10. Ding ZC, Lin YK, Gan YK, Tang TT. Molecular pathogenesis of fracture nonunion. J Orthop Translat. 2018;14:45-56.

11. Schmal H, Brix M, Bue M, Ekman A, Ferreira N, Gottlieb H, et al. Nonunion - consensus from the 4th annual meeting of the Danish Orthopaedic Trauma Society. EFORT Open Rev. 2020;5(1):46-57.

\section{Figures}

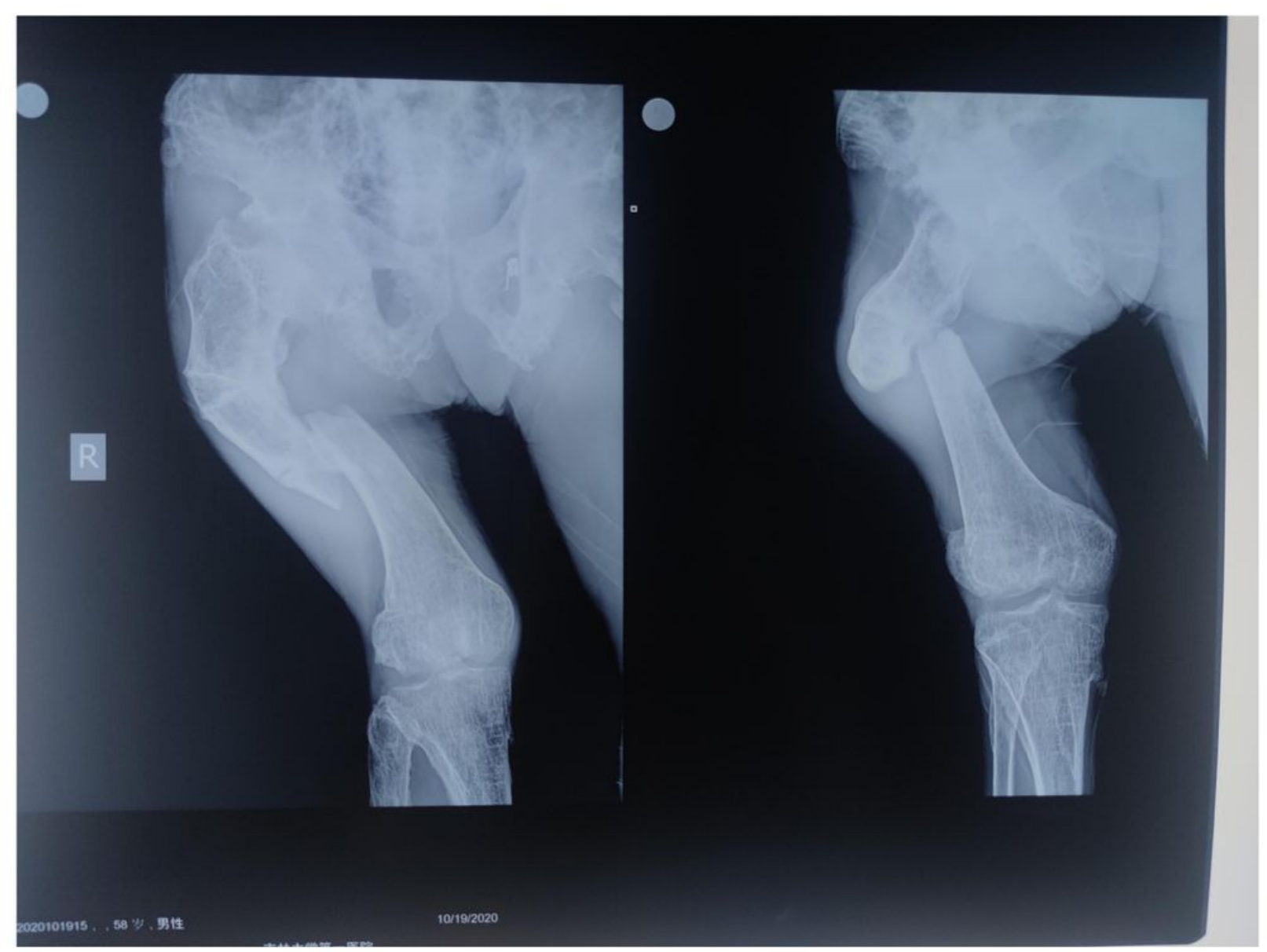

\section{Figure 1}


X-ray of the patient on admission

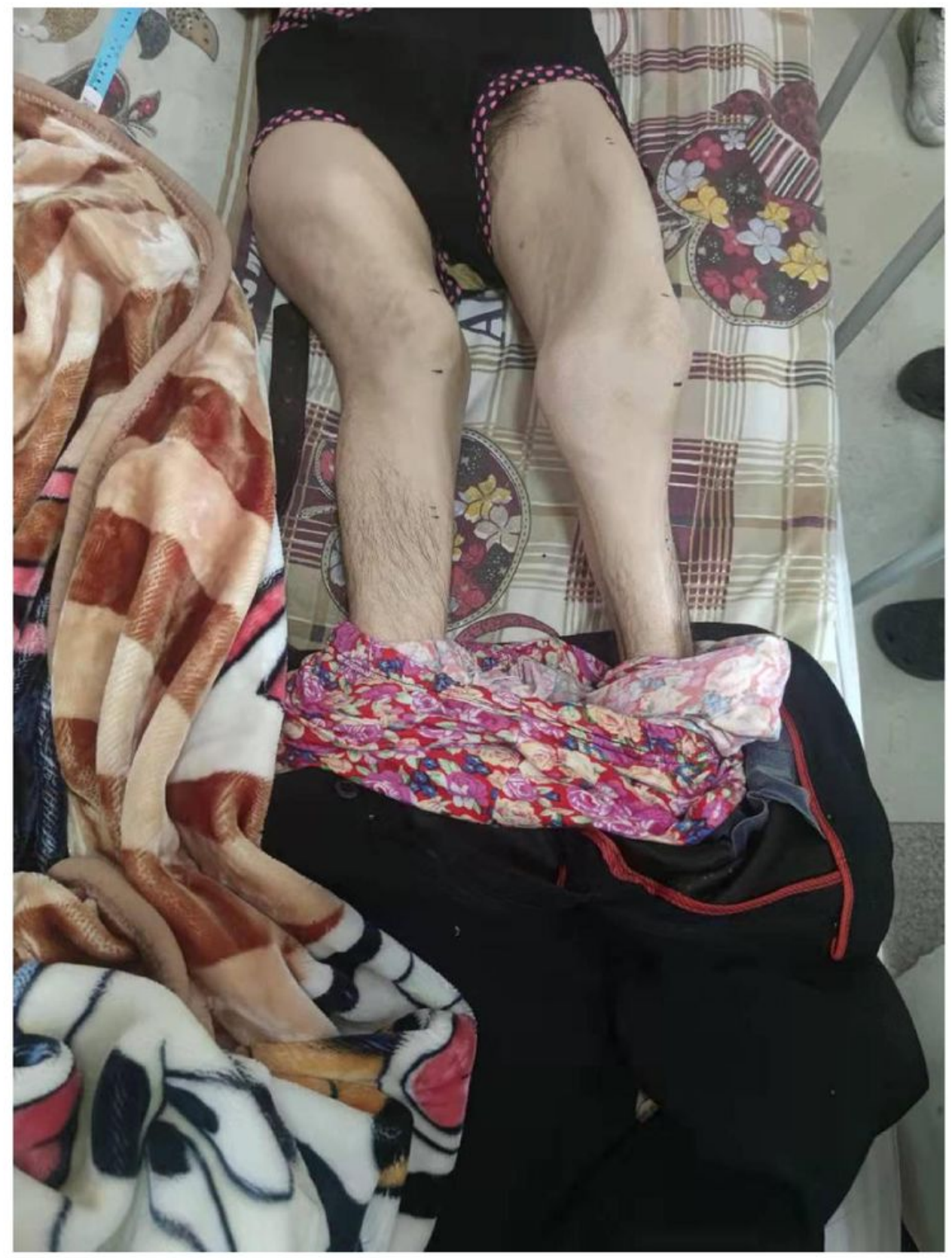

Figure 2

Preoperative external image 


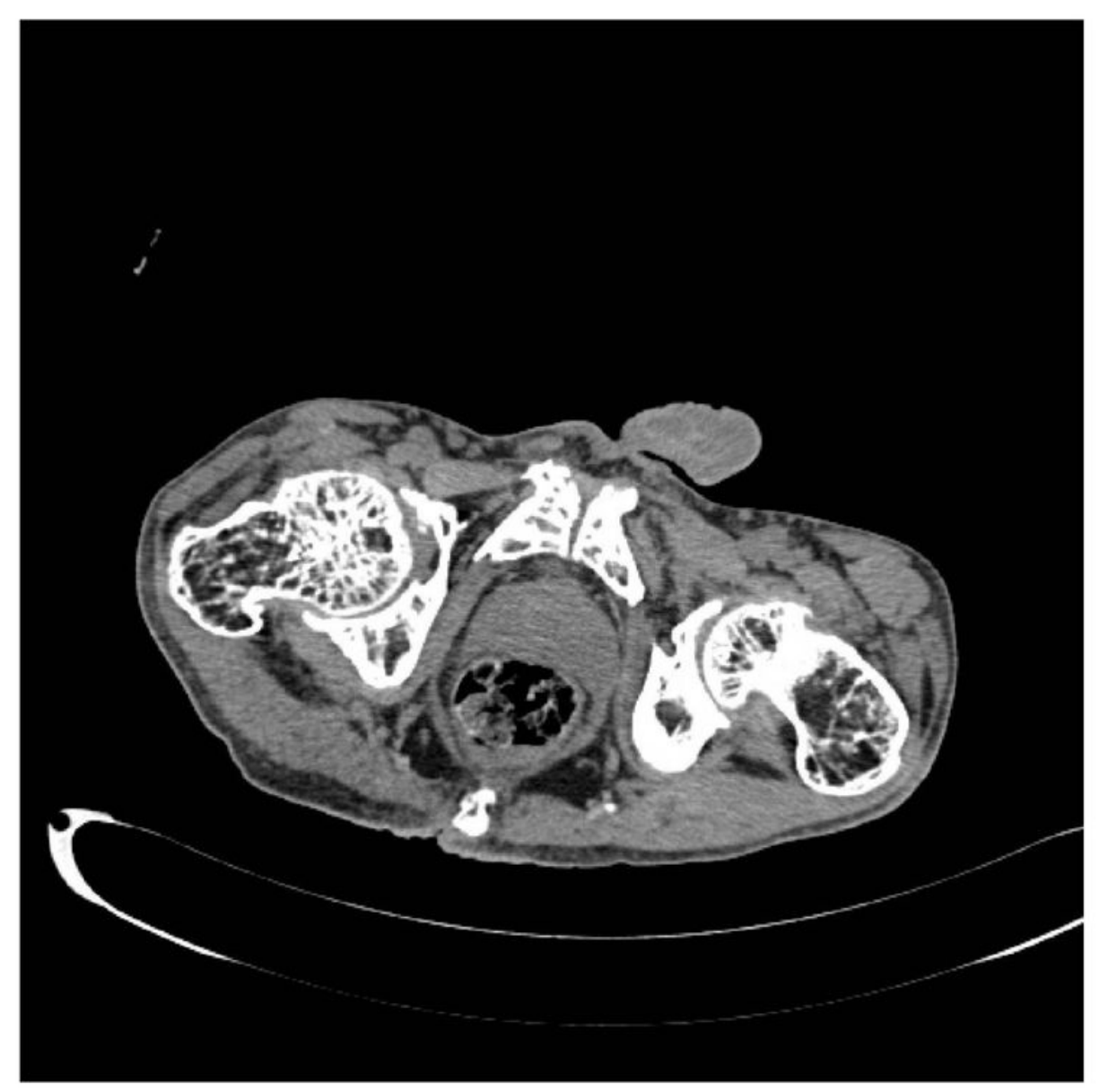

Figure 3

Preoperative CT 


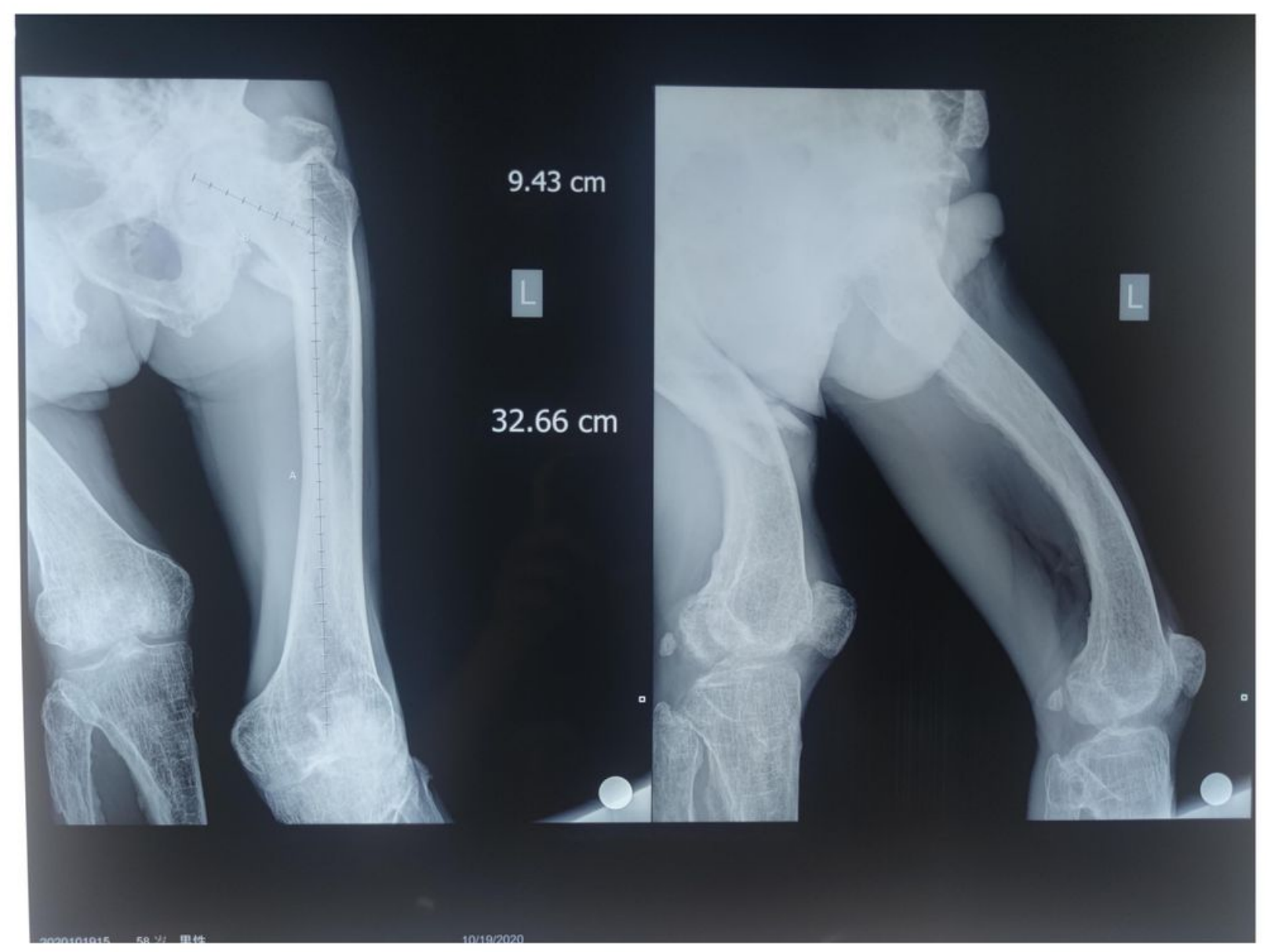

Figure 4

Preoperative imaging of the unaffected femur 


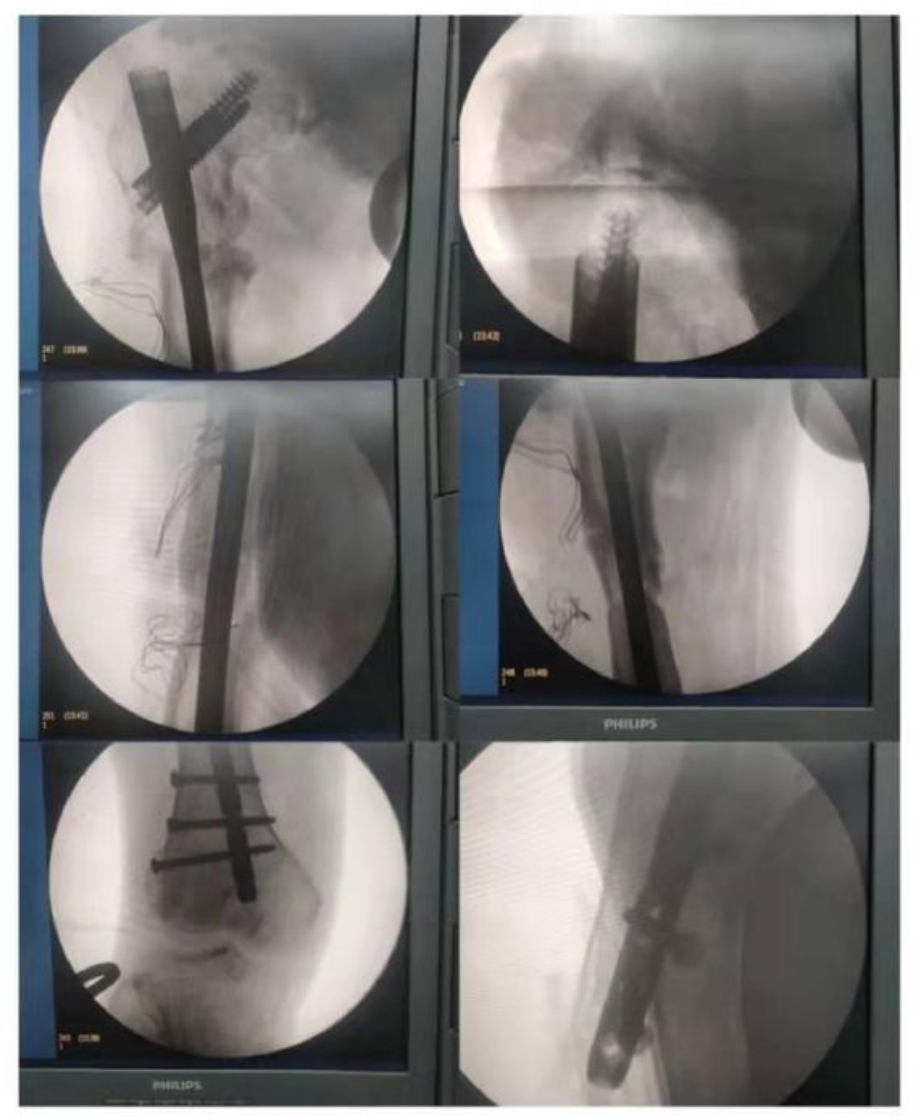

Figure 5

Intraoperative fluoroscopic imaging 


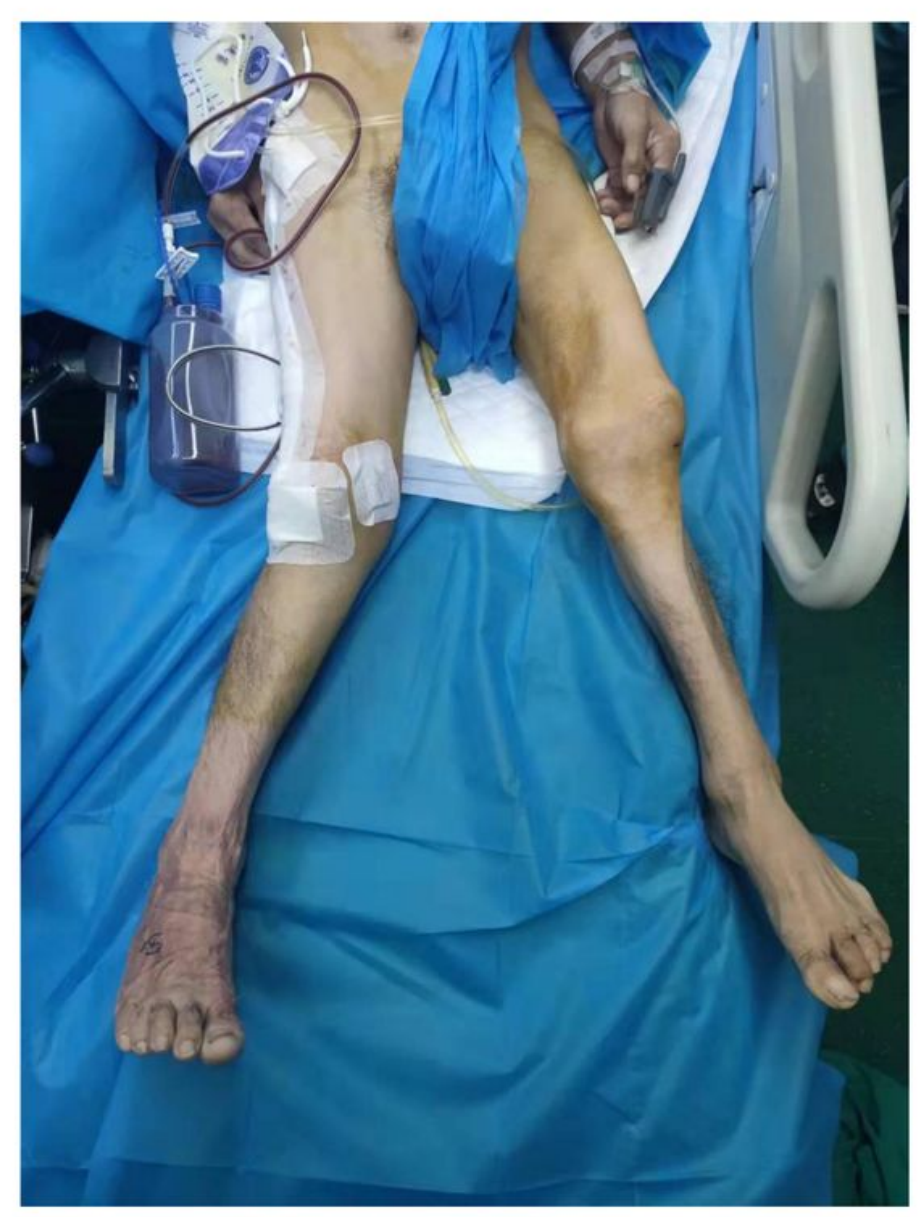

Figure 6

Postoperative external image 


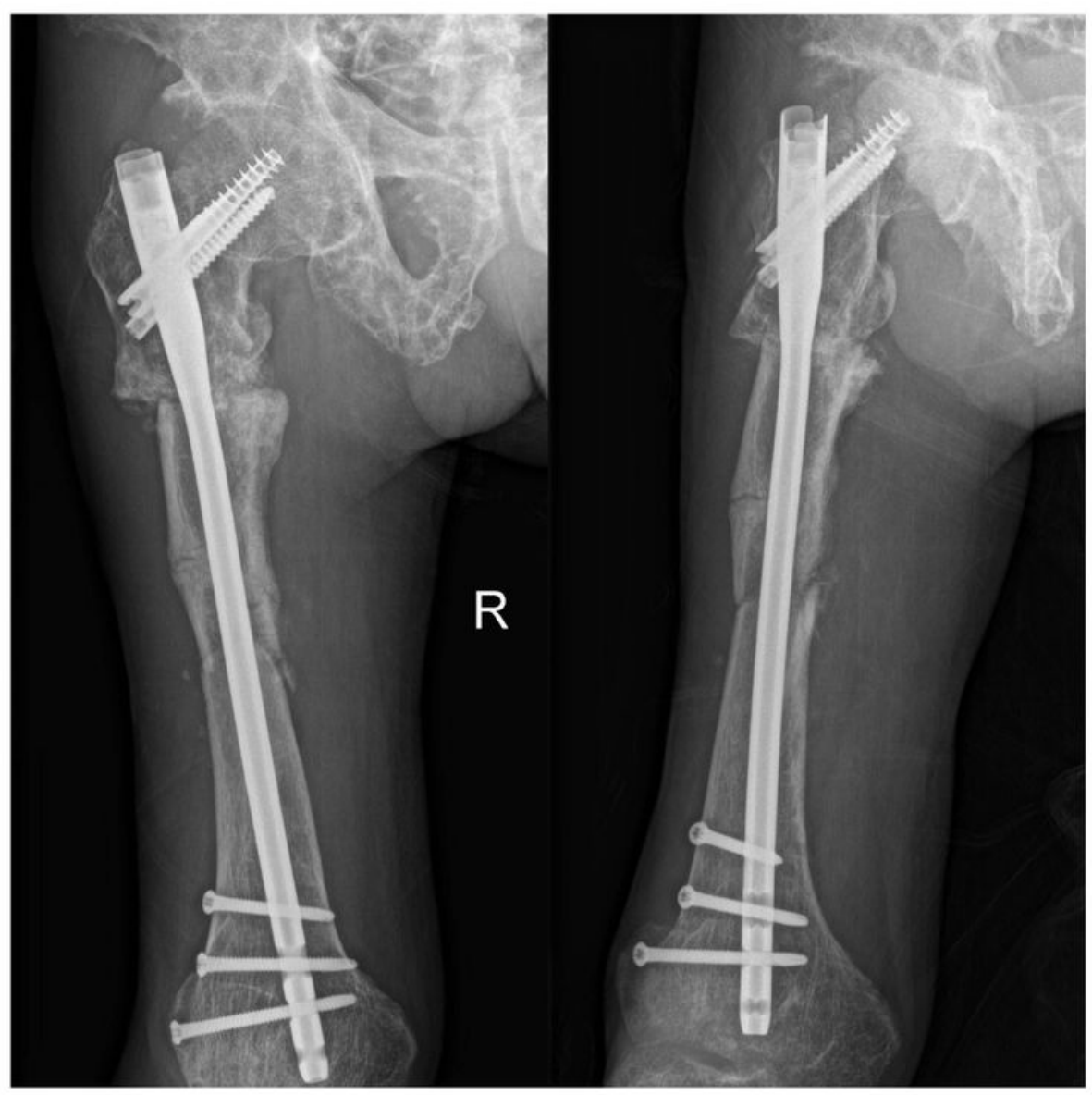

Figure 7

Follow-up 3 months after surgery 


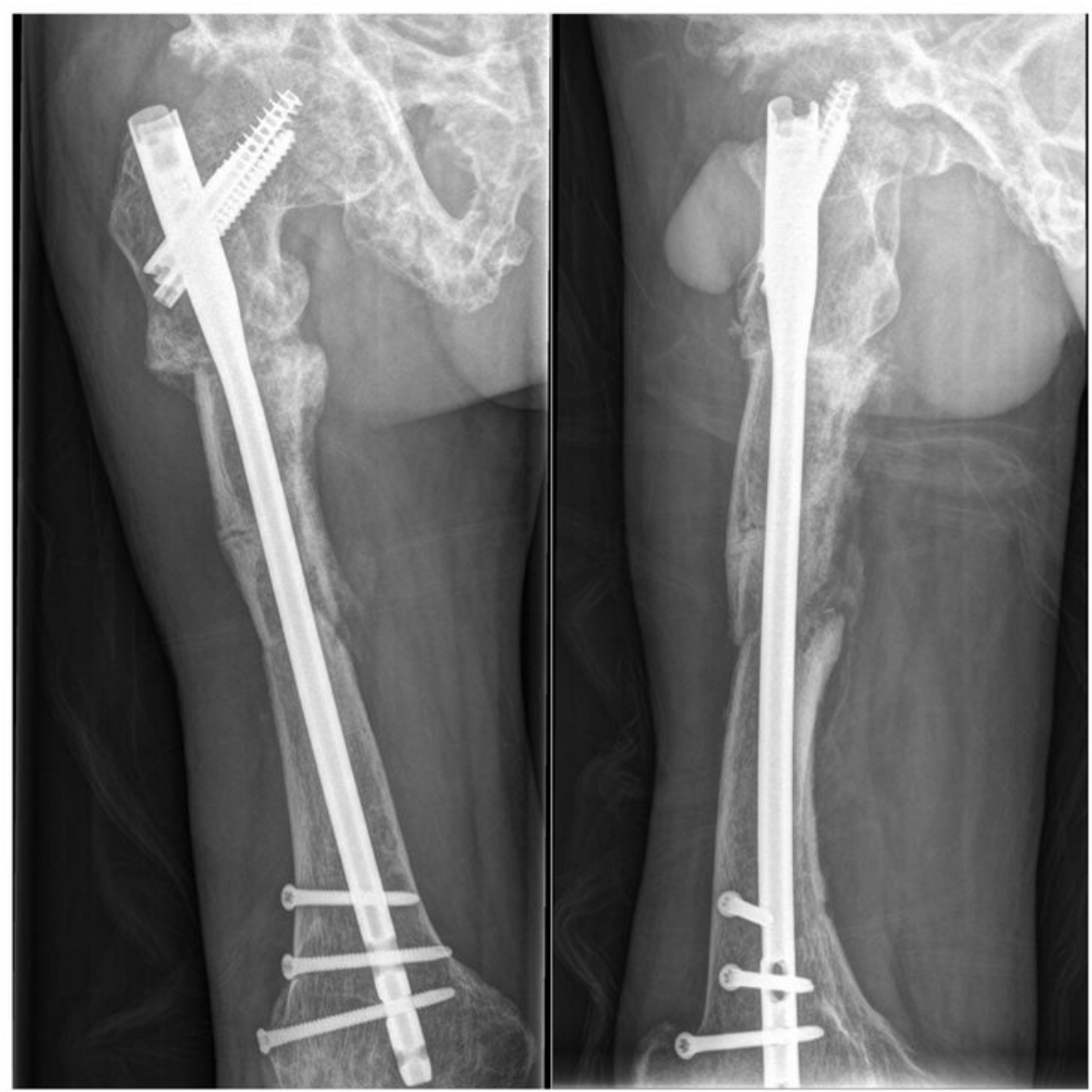

Figure 8

months after the operation, the fracture line was further 


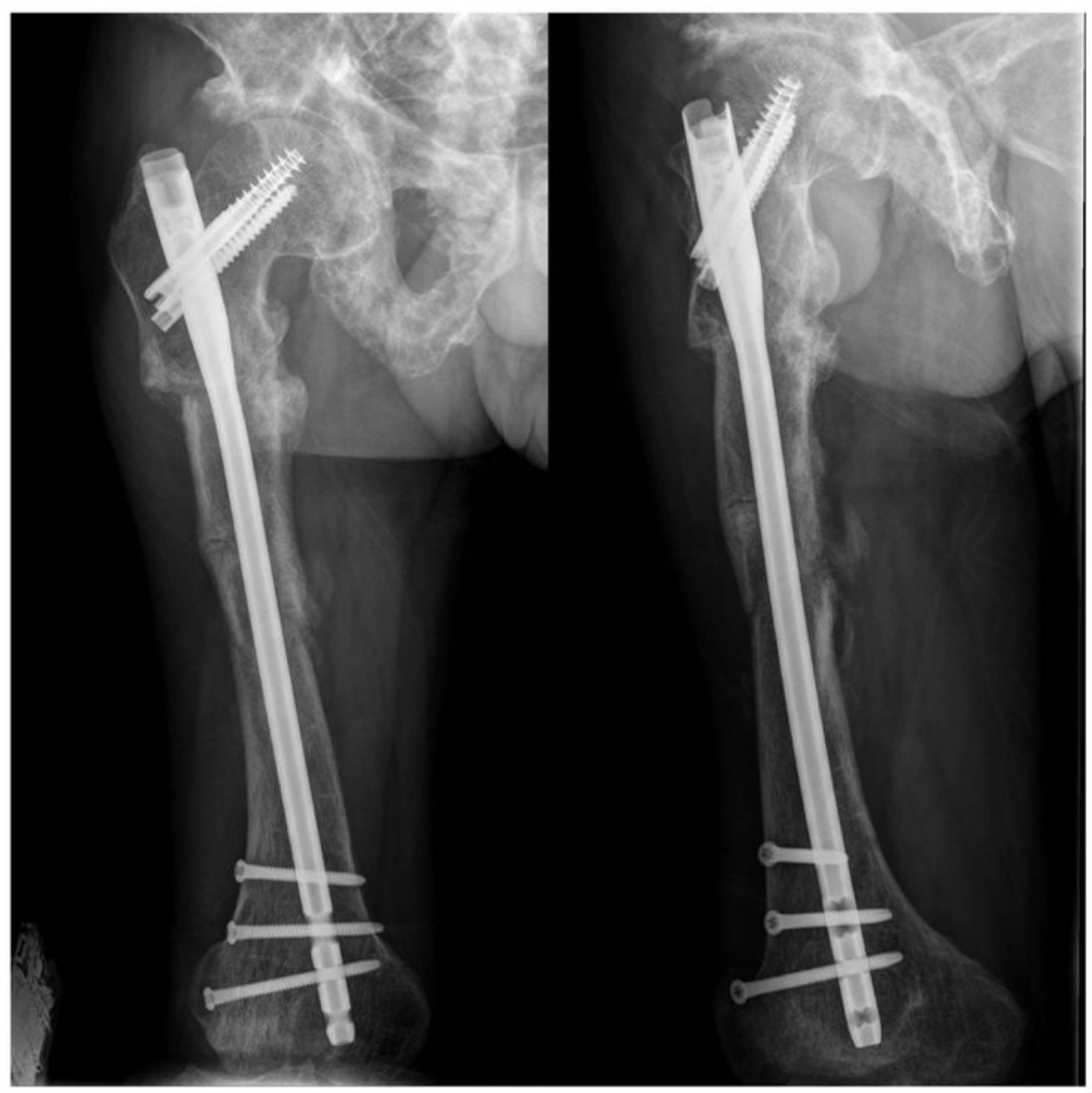

Figure 9

At 14 months postoperatively, the fracture was completely 


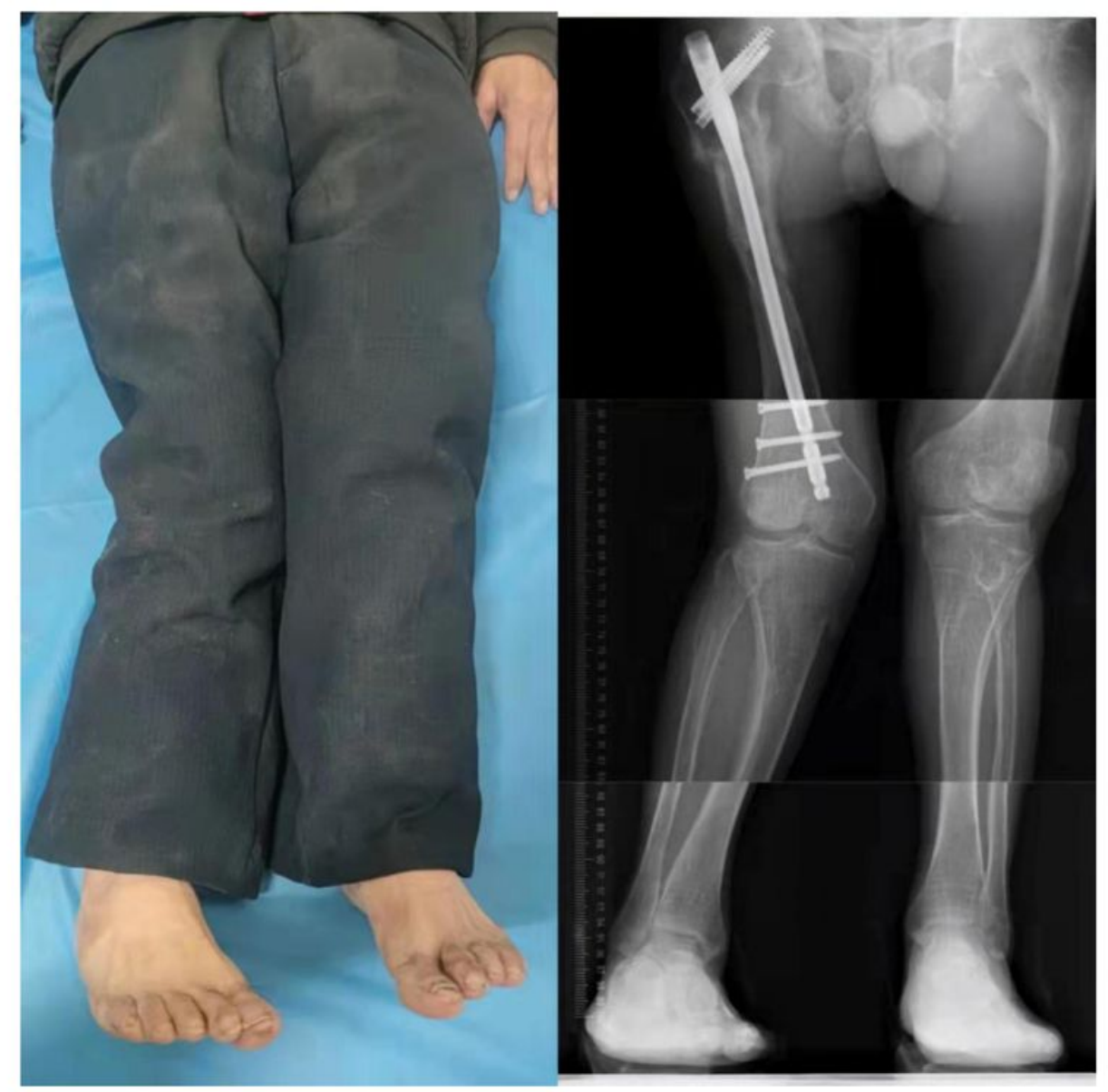

Figure 10

One-year postoperative image

\section{Supplementary Files}

This is a list of supplementary files associated with this preprint. Click to download.

- Patientwalkingvideo.mp4 